\title{
Circle of Willis: anatomical variations of configuration. A magnetic resonance angiography study
}

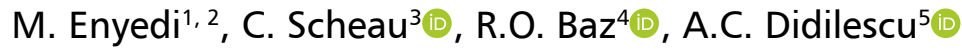 \\ ${ }^{1}$ Department of Anatomy, Carol Davila University of Medicine and Pharmacy, Bucharest, Romania \\ 2"Victor Babes" Centre for Diagnosis and Treatment, Bucharest, Romania \\ ${ }^{3}$ Department of Physiology, Carol Davila University of Medicine and Pharmacy, Bucharest, Romania \\ ${ }^{4}$ Department of Radiology, County Clinical Emergency Hospital, Constanța, Romania \\ ${ }^{5}$ Department of Embryology, Faculty of Dental Medicine, Carol Davila University of Medicine and Pharmacy, \\ Bucharest, Romania
}

[Received: 4 October 2021; Accepted: 30 November 2021; Early publication date: 15 December 2021]

Background: The main scope of this paper is to investigate the prevalence of the anatomical variants of the circle of Willis (CoW) in the Romanian population through magnetic resonance angiography.

Materials and methods: Magnetic resonance angiography images were obtained for 126 individuals and the configurations of the anterior and posterior CoW were identified, and classified. The prevalence of each variant and the number of complete anterior or posterior parts of the circle were determined.

Results: A classical configuration of the CoW was found in 39 (30.9\%) cases. The most common posterior variation was the unilateral absence of a posterior communicating artery $(n=28)$ while in the anterior circle it was the unilateral absence of the precommunicating segment of an anterior cerebral artery $(n=17)$. A complete entire CoW was found in 63 cases, while the anterior and posterior parts yielded complete configurations in 108 and 73 cases, respectively. Eight cases did not present complete configurations. A foetal posterior communicating artery was identified unilaterally in 14 cases and bilaterally in 6 cases.

Conclusions: Unilateral variations were the most common changes found in CoW configuration. The correct assessment of the CoW configuration may prove useful in the planning and follow-up of brain surgery and interventional procedures, as well as in estimating the prognosis of patients suffering from stroke or other related cerebral vascular events. (Folia Morphol 2023; 82, 1: 24-29)

Key words: circle of Willis, anatomic variation, magnetic resonance angiography

\section{INTRODUCTION}

The circle of Willis (CoW) is defined as the ring-shaped vascular structure situated at the base of the brain, where the anterior (internal carotid) and posterior (vertebral) circulations converge. It is named after the anatomist Thomas Willis who vastly contributed to its understanding, and is also called the

Address for correspondence: C. Scheau, MD, PhD, 8 Eroii Sanitari Blvd., 5th district, 050474, Bucharest, Romania, tel: +40213103722 , e-mail: cristian.scheau@umfcd.ro

This article is available in open access under Creative Common Attribution-Non-Commercial-No Derivatives 4.0 International (CC BY-NC-ND 4.0) license, allowing to download articles and share them with others as long as they credit the authors and the publisher, but without permission to change them in any way or use them commercially. 
cerebral arterial circle, the Willis polygon, and the loop of Willis [22].

One of the most important functions of the CoW is to sustain the blood flow through its network of anastomoses, in case of disturbances involving one of its branches [11]. When certain conditions are met, the particular configuration of the CoW provides a fail-safe, maintaing the brain tissue vascularisation by rerouting the cerebral haemodynamics [1]. Howev$\mathrm{er}$, in the case of an anatomical variant of the CoW that yields an incomplete circle, the compensatory capacity is lost, and severe flow hindering in either internal carotid or vertebral artery leads to ischaemia and potentially exitus [2]. Thus, knowledge of the configuration of the CoW is extremely important in patients undergoing cerebral vascular surgery where one of the branches may be involved, as well as in patients with chronic vascular diseases leading to occlusions.

Studies on various world populations cite an incidence between $16.6 \%$ and $85.4 \%$ for the "classical" configuration of the CoW [12, 21, 23-25]. Significant differences between populations of various races were noted, and genetic and environmental factors were considered as elements that may influence the development of the CoW $[10,16,21,23$, 24]. This paper aims to investigate the anatomical variants of the CoW and to compare their prevalence to the available literature studies. To our knowledge, this is the first report on the CoW configurations in a Romanian cohort.

\section{MATERIALS AND METHODS}

\section{Study design}

We designed a retrospective cohort study that included all patients that were submitted to cerebral magnetic resonance angiography (MRA) in a defined period of time, recording the configurations of the CoW in all examinations.

The imaging studies were performed in the "Victor Babes" Centre for Diagnosis and Treatment, Bucharest, Romania, between 01.05.2019 and 30.09.2019. All cerebral investigations that included the MRA sequence were considered. The data is stored in the local picture archiving and communication system, for an indefinite period of time. No follow-up was performed.

All configurations of the anterior and posterior parts of the CoW were recorded and classified based on the model of distribution proposed by Chen et al. [6] which proposes 10 anterior and 10 posterior patterns of the circle. According to this classification, the following aspects are mentionable: unilateral fetal origin of the posterior cerebral artery (fPCA) corresponds to posterior circle variants $B, F, G$ and $H$, while the bilateral fPCA is found in variants $C, I$ and $J$; also, a complete anterior circle is observed in variants A through $\mathrm{F}$, while the posterior circle is complete in variants $A$ through $C$; a complete entire circle requires concurring complete anterior and posterior circles. The imaging assessment regarded the topography, relative diameter and morphology of the internal carotid arteries (ICA), anterior, middle and posterior cerebral arteries (ACA, MCA and PCA, respectively), as well as the anterior and posterior communicating arteries (ACOA and $\mathrm{PCOA}$ ). Other measured variables were the patients' ages and gender.

\section{Participants}

The study population consisted of 126 patients (53 male, 73 female), aged between 1 and 93 years (mean age of $36 \pm 24$ years), that underwent cerebral MRA in our clinic for developmental delay (for the 14 patients aged 1 to 5 years old) or non-specific neurological symptoms including headache, dizziness, nausea, and anxiety. Patients with prior brain surgery or interventional procedures were excluded.

Informed consent for the imaging investigation, as well as for the processing and presenting of data, was obtained from all patients or guardians, respectively. The study was approved by the institutional ethics committee (registration no.: 1523/02.04.2020) and was performed in accordance with the Declaration of Helsinki and its later amendments.

\section{Image acquisition}

The images were acquired on a Siemens MAGNETOM Avanto 1.5 Tesla using the dedicated 12 element Head Matrix coil. The MRA protocol employed in all cases relied on the three-dimensional-time-of-flight (3D-TOF) imaging method (repetition time: $25 \mathrm{~ms}$, echo time: $7 \mathrm{~ms}$, resolution of $0.7 \times 0.7 \times$ $\times 0.7 \mathrm{~mm}$ with a $180 \times 180 \mathrm{~mm}$ field of view). The imaging interpretation was performed on a dedicated workstation and the CoW variants were recorded for each patient, for the anterior and posterior components. An arterial vessel was deemed absent when it was not visualised and hypoplastic when its diameter was smaller than $0.8 \mathrm{~mm}$. The 3D-TOF sequence was repeated when motion or other artefacts occurred in order to obtain a satisfactory image quality. There 
was no need to exclude investigations due to motion or metallic artefacts, thus the study size included all investigations available in the three months of recruiting.

\section{Ethics approval and consent to participate}

Institutional Ethics Committee approval was obtained (id no: 1523/02.04.2020).

All patients (or caregivers, or guardians when applicable) gave their informed consent to be submitted to the investigation and for their data to be processed and used in research purposes.

\section{Statistical methods}

Data distributions were expressed as means, standard deviations and percentages. Possible associations between gender and variant types were tested using Pearson chi $^{2}$ test. Fisher's exact test was used when the expected frequency in any cell was less than 5 . The level of significance was set at 0.05 . We performed statistical analyses using Stata/IC 16 (StataCorp).

\section{RESULTS}

The prevalence of the CoW anatomic variants is presented in Tables 1 and 2, for the anterior and posterior parts, respectively. While the model used for classification yields 10 possible variants for each of the two parts of the CoW, we only encountered 8 variants in the anterior ( $A$ through $H)$, and 6 variants in the posterior CoW (A through $\mathrm{F}$ ); example cases of all identified anterior and posterior configurations are presented in Figure 1.

A classical configuration of the CoW was found in $39(30.9 \%)$ cases. The most common variation from the classical configurations was the unilateral absence of one of the posterior communicating arteries $(n=28)$, which was also the most frequent variation of the posterior circle. The most common variation in the anterior part of the CoW was the unilateral absence of the precommunicating segment of an anterior cerebral artery $(n=17)$.

A statistical association between the female gender and the presence of a bilateral PPCA (posterior CoW variant $C$ ) was identified ( $p=0.039$ ).

The prevalence of the complete anterior, posterior and entire CoW is presented in Table 3. A complete structure of the entire CoW was found in 63 cases, while the anterior and posterior parts yielded complete configurations in 108 and 73 cases, respectively.
Table 1. The prevalence of the anatomic variants of the anterior circle of Willis in relation to patient gender

\begin{tabular}{lcccccccc}
\hline Group & \multicolumn{7}{c}{ Variant type (\%) } \\
\cline { 2 - 9 } & A & B & C & D & E & F & G & H \\
\hline Males & $64.2 \%$ & 0 & $9.4 \%$ & 0 & $1.9 \%$ & $9.4 \%$ & $5.7 \%$ & $13.2 \%$ \\
Females & $68.5 \%$ & $2.8 \%$ & $6.9 \%$ & $1.4 \%$ & $1.4 \%$ & $5.5 \%$ & $2.7 \%$ & $13.7 \%$ \\
Total & $66.7 \%$ & $1.6 \%$ & $7.9 \%$ & $0.8 \%$ & $1.6 \%$ & $7.1 \%$ & $4 \%$ & $13.5 \%$ \\
\hline
\end{tabular}

Table 2. The prevalence of the anatomic variants of the posterior circle of Willis in relation to patient gender

\begin{tabular}{lcccccc}
\hline \multirow{2}{*}{ Group } & \multicolumn{6}{c}{ Variant type (\%) } \\
\cline { 2 - 7 } & A & B & C & D & E & F \\
\hline Males & $52.8 \%$ & $7.6 \%$ & 0 & $24.5 \%$ & $11.3 \%$ & $1.9 \%$ \\
Females & $39.7 \%$ & $8.2 \%$ & $8.2 \% *$ & $20.6 \%$ & $19.2 \%$ & $4.1 \%$ \\
Total & $45.2 \%$ & $7.9 \%$ & $4.8 \%$ & $22.2 \%$ & $15.9 \%$ & $3.2 \%$ \\
\hline
\end{tabular}

${ }^{*} p=0.039$, Fisher's exact test

Eight (6.4\%) cases did not present complete configurations neither in anterior, nor in posterior CoW. Among them, 50\% were males.

The bilateral $\mathrm{APCA}$ presence was identified in a total of 6 cases, while a unilateral PPCA was detected in 14 cases. The gender-relative prevalence for the adult and fetal configurations of the CoW is depicted in Table 4.

No incidental aneurysms were identified in the CoW vessels in the study group.

\section{DISCUSSION}

Despite diverse classifications of the CoW variations are available, the present study relied on the one proposed by Chen et al. [6], which we found to be the most simple and intuitive, while also addressing the complexity and clinical relevance of the different configurations $[6,9,13,15,17]$. Moreover, the existence of multiple similar papers that have adopted this method in various populations was encouraging in regard to presenting data in a manner that allows for comparison in future studies. Out of the 20 total possible configurations of the CoW, we identified only the first 8 for the anterior, and first 6 for the posterior circle, respectively. A scarcity of these unidentified variants was cited in other similar studies with comparable study population size [25].

The possibility of a correlation between the configuration of the CoW and the type of ethnic or racial group is a recurring conception and was one of the fundamental grounds for our paper, alongside the 

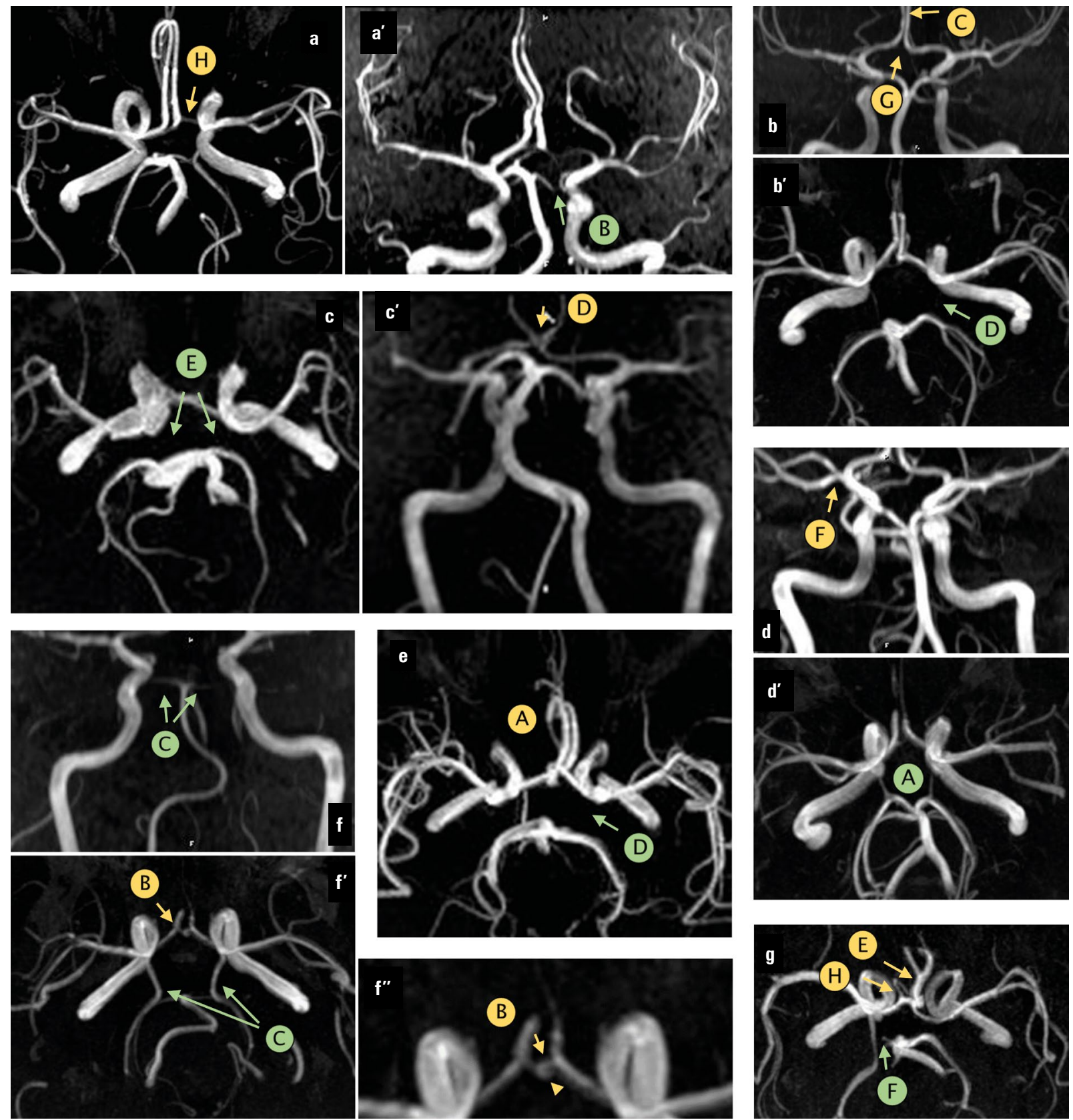

Figure 1. A collection of maximum intensity projection images of three-dimensional-time-of-flight acquisitions from 7 cases from the study group (labelled a through f). The anatomic variants of the anterior (yellow) and posterior (green) circle of Willis (CoW) are represented with arrows, except for the type A configuration, which is identified in the anterior (e) and posterior (d') components, respectively. For the anterior CoW, the anatomic variants depicted are, as follows: duplication of the anterior communicating arteries (ACoA) in the axial plane in images $f^{\prime}$ and detailed in $\mathbf{f}^{\prime \prime}$ (arrow and arrowhead); medial artery of the corpus callosum originating in the ACoA (b); fusion of post-communicating anterior cerebral artery (ACAs) (c'); common trunk for post-communicating ACAs (g); double trunk of middle cerebral artery (d); absent $\mathrm{ACoA}(\mathbf{b})$; hypoplastic precommunicating ACA (a). In the posterior CoW we identified: unilateral posterior cerebral artery (fPCA) (a'), bilateral fPCA (f, $\left.\mathbf{f}^{\prime}\right)$, unilateral absent posterior communicating artery (PCoA) (e), bilateral absent PCoA (c), unilateral fetal type PCA with absent precommunicating segment $(\mathbf{g})$.

paucity of reports regarding the configuration of the CoW in the Romanian population [9]. However, we did not perform a thorough comparison to reports from other populations, as it did not fall within the scope of our study, and we consider that a structured/ /systematic review is more appropriate in order to draw relevant conclusions on this topic.

The development of the CoW in the embryo occurs in two stages: vasculogenesis and angiogenesis; the events occurring in the embryological state can lead 
Table 3. The prevalence of the complete anterior, posterior and entire circle of Willis (CoW) in relation to patient gender

\begin{tabular}{lccc}
\hline Group & $\begin{array}{c}\text { Complete ante- } \\
\text { rior CoW (\%) }\end{array}$ & $\begin{array}{c}\text { Complete pos- } \\
\text { terior CoW (\%) }\end{array}$ & $\begin{array}{c}\text { Complete entire } \\
\text { CoW (\%) }\end{array}$ \\
\hline Males & $84.9 \%$ & $60.4 \%$ & $52.8 \%$ \\
Females & $86.3 \%$ & $56.2 \%$ & $47.9 \%$ \\
Total & $85.7 \%$ & $57.9 \%$ & $50 \%$ \\
\hline
\end{tabular}

Table 4. The prevalence of the adult and fetal circle of Willis (CoW) in relation to patient gender

\begin{tabular}{lccc}
\hline Group & $\begin{array}{c}\text { Adult } \\
\text { CoW (\%) }\end{array}$ & $\begin{array}{c}\text { Fetal unilateral } \\
\text { CoW (\%) }\end{array}$ & $\begin{array}{c}\text { Fetal total (uni + } \\
\text { + bilateral) CoW (\%) }\end{array}$ \\
\hline Males & $88.7 \%$ & $9.4 \%$ & $9.4 \%$ \\
Females & $79.5 \%$ & $12.3 \%$ & $20.6 \%$ \\
Total & $83.3 \%$ & $11.1 \%$ & $15.9 \%$ \\
\hline
\end{tabular}

to either anomalies of normal arteries or the persistence of embryonic arteries or anastomoses [20].

A "classical" configuration with a complete entire circle and "standard" configuration of distribution and size of the arteries contributing to the CoW was found in $30.9 \%$ of cases, which is within the range of most similar studies $[23,25]$. The most common variation from the classical aspect was identified as the unilateral absence of one of the posterior communicating arteries $(22.2 \%)$ in the posterior CoW, while in the anterior circle, the second most common configuration after the classical pattern was the unilateral absence of the precommunicating segment of an anterior cerebral artery (13.5\%).

The complete entire circle was identified in $50 \%$ of subjects, which is analogous to other similar studies, despite some reports that cite a very high prevalence of up to $99.4 \%$ complete circles $[3,14]$. Eight $(6.4 \%)$ cases presented an incomplete anterior and posterior circle, which may represent a risk factor for a cardiovascular event, as this condition was linked to poorer prognosis and higher severity of strokes [27]. A complete CoW was found to be not only a protective factor in stroke, but also a predictor of a more favourable clinical outcome in stroke, with less haemorrhage transformation [26].

A bilateral fPCA was identified statistically significant more common in women than in men; however, the correlation may be incidental and relevance of this finding should be validated in larger studies.

Adult type circulation was found in 105 (83.3\%) patients, with fetal circulation identified unilaterally in $11.1 \%$ of cases, and bilaterally in $5.6 \%$ of cases, a finding that is congruent with literature studies [15, $19,24]$. In the evaluation of adult vs. fetal type circulation, that relies on deciding whether the posterior communicating artery is larger than the precommunicating segment of the posterior cerebral artery, some studies cite a third variant called transitional, where the two arteries are identical in size; however, we did not take this option into consideration, as we considered that it can introduce bias due to the limits in accurately comparing the vessel diameters, where the imaging plane, resolution and scale, blood flow, and imaging software may influence the measurements.

Differences in prevalence of CoW configuration were noted between imaging and autopsy studies [16]. Possible contributing factors are, among others, the cutoff size value for considering a vessel hypoplastic, flow turbulences and artefacts that may impede correct assessment on imaging studies, and operator-dependent limits in the cadaveric dissection.

This study has limitations, the most important being the study population size, that may not accurately describe the distribution of the CoW configuration in the general population. Imaging artefacts may hinder the correct assigning of the variants; however, no such distortions were identified. Nevertheless, while MRA is a very competent method in evaluating the CoW configuration and the patency of any of its branches and has undergone significant technical improvements over the recent years, conventional angiography is still regarded by some authors as the gold standard in specific cases $[7,8]$.

The adequate imaging analysis of vessel morphology may uncover the presence of inflammatory or tumoural lesions and may contribute to a superior understanding of vascular dynamics, greatly impacting the management of selected patients $[4,5,18]$.

\section{CONCLUSIONS}

To our knowledge, this is the first paper that assesses the configuration variants of the CoW through MRA in our country. The findings may prove useful in the planning and follow-up of brain surgery and interventional procedures, as well as in estimating the prognosis of patients suffering from stroke or other related cerebral vascular events.

\section{Conflict of interest: None declared}

\section{REFERENCES}

1. AbuRahma AF, Mousa AY, Stone PA, et al. Correlation of intraoperative collateral perfusion pressure during carotid 
endarterectomy and status of the contralateral carotid artery and collateral cerebral blood flow. Ann Vasc Surg. 2011; 25(6): 830-836, doi: 10.1016/j.avsg.2011.04.002, indexed in Pubmed: 21680143.

2. Alastruey J, Parker KH, Peiró J, et al. Modelling the circle of Willis to assess the effects of anatomical variations and occlusions on cerebral flows. J Biomech. 2007; 40(8): 1794-1805, doi: 10.1016/j.jbiomech.2006.07.008, indexed in Pubmed: 17045276.

3. Alpers BJ, Berry RG, Paddison RM. Anatomical studies of the circle of Willis in normal brain. AMA Arch Neurol Psychiatry. 1959; 81(4): 409-418, doi: 10.1001/archneurpsyc.1959.02340160007002, indexed in Pubmed: 13636509.

4. Baz RA, Scheau C, Niscoveanu C, et al. Morphometry of the entire internal carotid artery on CT angiography. Medicina (Kaunas). 2021; 57(8), doi: 10.3390/medicina57080832, indexed in Pubmed: 34441039.

5. Baz RA, Scheau C, Sârbu N, et al. Carotid paragangliomas: case report and imaging review. Folia Morphol. 2021; 80(3): 699-706, doi: 10.5603/FM.a2020.0078, indexed in Pubmed: 32710793.

6. Chen HW, Yen PS, Lee CC, et al. Magnetic resonance angiographic evaluation of circle of Willis in general population: A morphologic study in 507 cases. Chin J Radiol. 2004; 29: 223-229.

7. Cuong NN, Luu VD, Tuan TA, et al. Conventional digital subtractional vs non-invasive MR angiography in the assessment of brain arteriovenous malformation. Clin Neurol Neurosurg. 2018; 169: 29-33, doi: 10.1016/j. clineuro.2018.03.022, indexed in Pubmed: 29604508.

8. D'Arco F, D'Amico A, Caranci F, et al. Cerebrovascular stenosis in neurofibromatosis type 1 and utility of magnetic resonance angiography: our experience and literature review. Radiol Med. 2014; 119(6): 415-421, doi: 10.1007/s11547-013-0358-8, indexed in Pubmed: 24297593.

9. Eftekhar B, Dadmehr M, Ansari S, et al. Are the distributions of variations of circle of Willis different in different populations? Results of an anatomical study and review of literature. BMC Neurol. 2006; 6: 22, doi: 10.1186/14712377-6-22, indexed in Pubmed: 16796761.

10. Forgo B, Tarnoki AD, Tarnoki DL, et al. Investigation of circle of Willis variants and hemodynamic parameters in twins using transcranial color-coded Doppler sonography. Int J Cardiovasc Imaging. 2018; 34(9): 1419-1427, doi: 10.1007/ s10554-018-1359-4, indexed in Pubmed: 29675634.

11. Jahed M, Ghalichi F, Farhoudi M. Comparison of blood velocity between transcranial Doppler and numerical method in the patient-specific circle of Willis with aneurysm. Biomed Mater Eng. 2019; 30(4): 427-438, doi: 10.3233/ BME-191064, indexed in Pubmed: 31561321.

12. Jalali Kondori B, Azemati F, Dadseresht S. Magnetic resonance angiographic study of anatomic variations of the circle of willis in a population in Tehran. Arch Iran Med. 2017; 20(4): 235-239, doi: 0172004/aim.009, indexed in Pubmed: 28412828.

13. Jin ZN, Dong WT, Cai XW, et al. CTA characteristics of the circle of Willis and intracranial aneurysm in a Chinese crowd with family history of stroke. Biomed Res Int. 2016; 2016: 1743794, doi: 10.1155/2016/1743794, indexed in Pubmed: 26881211.
14. Kapoor K, Singh B, Dewan LI. Variations in the configuration of the circle of Willis. Anat Sci Int. 2008; 83(2): 96-106, doi: 10.1111/j.1447-073X.2007.00216.x, indexed in Pubmed: 18507619.

15. Karatas A, Coban G, Cinar C, et al. Assessment of the circle of Willis with cranial tomography angiography. Med Sci Monit. 2015; 21: 2647-2652, doi: 10.12659/ MSM.894322, indexed in Pubmed: 26343887.

16. Klimek-Piotrowska W, Kopeć M, Kochana M, et al. Configurations of the circle of Willis: a computed tomography angiography based study on a Polish population. Folia Morphol. 2013; 72(4): 293-299, doi: 10.5603/ $\mathrm{fm} .2013 .0049$, indexed in Pubmed: 24402749.

17. Klimek-Piotrowska W, Rybicka M, Wojnarska A, et al. A multitude of variations in the configuration of the circle of Willis: an autopsy study. Anat Sci Int. 2016; 91(4): 325-333, doi: 10.1007/s12565-015-0301-2, indexed in Pubmed: 26439730.

18. Low A, Mak E, Rowe JB, et al. Inflammation and cerebral small vessel disease: a systematic review. Ageing Res Rev. 2019; 53: 100916, doi: 10.1016/j.arr.2019.100916, indexed in Pubmed: 31181331.

19. Machasio RM, Nyabanda R, Mutala TM. Proportion of variant anatomy of the circle of Willis and association with vascular anomalies on cerebral CT angiography. Radiol Res Pract. 2019; 2019: 6380801, doi: 10.1155/2019/6380801, indexed in Pubmed: 31316832.

20. Menshawi K, Mohr JP, Gutierrez J. A functional perspective on the embryology and anatomy of the cerebral blood supply. J Stroke. 2015; 17(2): 144-158, doi: 10.5853/ jos.2015.17.2.144, indexed in Pubmed: 26060802.

21. Naveen SR, Bhat V, Karthik GA. Magnetic resonance angiographic evaluation of circle of Willis: A morphologic study in a tertiary hospital set up. Ann Indian Acad Neurol. 2015; 18(4): 391-397, doi: 10.4103/0972-2327.165453, indexed in Pubmed: 26713008.

22. Rengachary SS, Xavier A, Manjila S, et al. The legendary contributions of Thomas Willis (1621-1675): the arterial circle and beyond. J Neurosurg. 2008; 109(4): 765-775, doi: 10.3171/ JNS/2008/109/10/0765, indexed in Pubmed: 18826368.

23. Siddiqi $\mathrm{H}$, Tahir M, Lone KP. Variations in cerebral arterial circle of Willis in adult Pakistani population. J Coll Physicians Surg Pak. 2013; 23(9): 615-619, doi: 09.2013/ jcpsp.615619, indexed in Pubmed: 24034183.

24. Yeniçeri IÖ, Çullu N, Deveer M, et al. Circle of Willis variations and artery diameter measurements in the Turkish population. Folia Morphol. 2017; 76(3): 420-425, doi: 10.5603/FM.a2017.0004, indexed in Pubmed: 28150270.

25. Zaki SM, Shaaban MH, Abd Al Galeel WA, et al. Configuration of the circle of Willis and its two parts among Egyptian: a magnetic resonance angiographic study. Folia Morphol. 2019; 78(4): 703-709, doi: 10.5603/ FM.a2019.0015, indexed in Pubmed: 30761512.

26. Zhao H, Wang B, Xu G, et al. Collateral grade of the Willis' circle predicts outcomes of acute intracranial internal carotid artery occlusion before thrombectomy. Brain Behav. 2019; 9(12): e01452, doi: 10.1002/brb3.1452, indexed in Pubmed: 31696661.

27. Zhou H, Sun J, Ji X, et al. Correlation between the integrity of the circle of Willis and the severity of initial noncardiac cerebral infarction and clinical prognosis. Medicine. 2016; 95(10): e2892, doi: 10.1097/MD.0000000000002892, indexed in Pubmed: 26962785. 